\title{
Chemical Abundances and Hierarchical Clustering
}

\author{
Tissera, P. B. \\ Institute of Astronomy and Space Science, Conicet, Argentina \\ Lambas, D. G. \\ IATE, Observatorio Astronómico Córdoba and Conicet, Argentina
}

\begin{abstract}
In this contribution we study the chemical enrichment of the interstellar medium and stellar population of the building blocks of current typical galaxies in the field, in cosmological hydrodynamics simulations. The simulations include detailed modeling of chemical enrichment by SNIa and SNII. In our simulations the missing metal problem is caused by chemical elements being locked up in stars, in the central regions (or bulges) mainly. Supernova energy feedback could help to reduce this concentration by expelling metals to the intergalactic medium.
\end{abstract}

We studied the chemical enrichment history of the structure in a hierarchical scenario by using a cosmological SPH code which includes detailed chemical enrichment by SNIa and SNII (Mosconi et al. 2001). In order to compare with available observational data we draw random lines-of-sight (LOS) through the building blocks of current typical galaxies in the field. We estimated the HI column densities and chemical abundances of different elements as a function of redshift. We studied the column densities that satisfied the observational criteria used for Damped Lyman $-\alpha$ Systems. Our results show that when mapped by random LOS the gaseous components in building blocks show the same level of metallicity enrichment $(\approx-0.30)$ and $\alpha$-enhancement as those estimated for the observed DLAs (Tissera et al. 2002; Cora et al. 2003). The star formation rate per unit area in the simulated DLAs are, on average, an order of magnitude lower than those of the whole system (Tissera \& Lambas 2004). Comparing the mean abundances of the gaseous components and the stellar populations along the LOS and those of the whole galactic systems, we found that the simulated DLAs predicted abundances that were off by $\approx 40-70 \%$. The missing metals are located in the central regions of the simulated galactic systems and mostly locked up in stars. These regions are mapped with a lower probability by LOS because of geometrical effects. Our results suggest that the missing metal problem (Pettini 2003) could be produced by chemical elements being locked in stars and in the central regions. However, we note that a SN driven galactic wind could be able to expell a significant fraction to the intergalactic medium reducing the problem. 


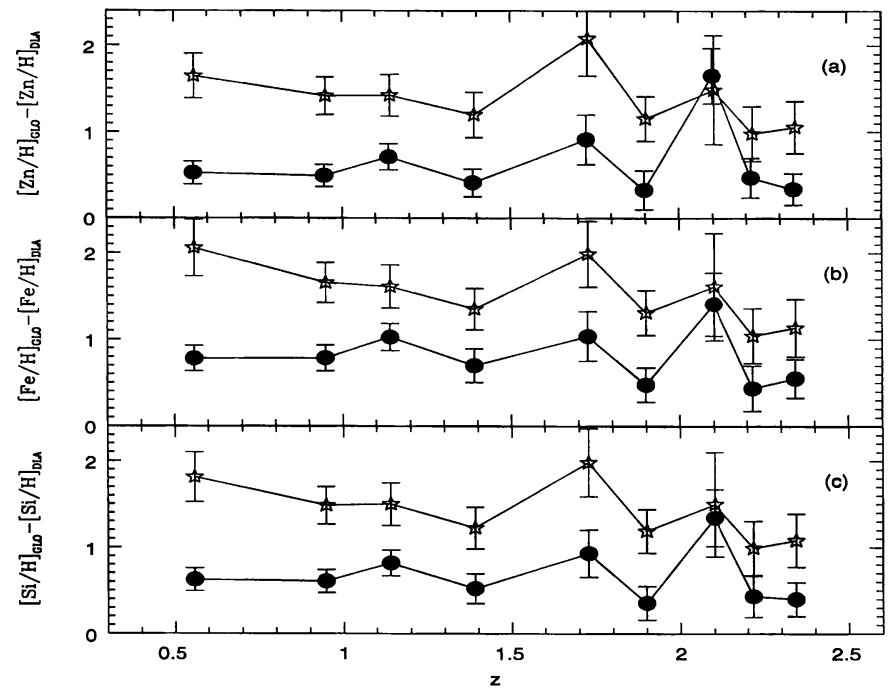

Figure 1. Averages of the differences between the mean abundances of the gaseous components (filled circles) and the stellar populations (open stars) in the simulated DLAs and those of their hostes systems as a function of redshift. Bootstrap errors shown.

\section{References}

Cora, S. A., Tissera, P. B., Lambas, D. G., \& Mosconi, M. 2003, MNRAS, 343, 959

Tissera, P. B., Lambas, D. G., Mosconi, M., \& Cora, S. A. 2002, MNRAS, 333, L27

Tissera, P. B., \& Lambas, D. G. 2004, MNRAS (submitted) 